Singh. D. Preference for bar pressing to obtain reward over freeloading in rats and children. Journal of Comparative \& Physiological Psychology, 1970, 73, 320-327.

Tarte, R. D.. \& Snyder, R. L. Bar pressing in the presence of free food as a function of food deprivation. Psychonomic Science, 1972, 26, 169-170.
Winer, B. J. Statistical principles in experimental design. New York: McGraw-Hill, 1971.

(Received for publication September 28, 1973.)

\title{
Recall of embedded sentences: Perceptual or performance deficit?*
}

\section{RAYMOND BAIRD \\ Wright State Universitu. Davton. Ohio 45431}

College students attempted verbatim recall or recognition of sentences containing embedded or nonembedded relative clauses. Embedded sentences were more difficult than nonembedded in verbatim recall, but the sentence types were equally difficult in the recognition task, implying that the grammatical relations and meaning of both sentence types are recovered and entered into memory.

Sentences containing embedded relative clauses have been found to be more difficult to recall than sentences of the same length containing an equal number of nonembedded clauses (Miller \& Isard, 1964; Baird, 1973). Baird found this to be true both when the embedded construction was nested within the matrix sentence, e.g.,

(1) The friend whom the lawyer whom the attendant painted soaked liked the teller

and when the embedded construction was appended to the matrix sentence, e.g.,

(2) The child bit the man whom the doctor who chased the dog liked

Both these types of construction, called nested/embedded (N/E) and nonnested/embedded $(\mathrm{NN} / \mathrm{E})$, respectively, were more difficult to recall then their corresponding nonembedded forms, e.g.,

(3) The cat who saw the girl who paid the cashier caught the passenger (N/NE)

(4) The attendant painted the lawyer who soaked the friend who liked the teller (NN/NE)

*The author wishes to thank Dale Brinkman, John Koslick, and Douglas Finch for their assistance in this series of studies. This paper is sponsored by George E. Briggs, who takes full editorial responsibility for its contents.
Conjecture concerning the psychological reasons for these findings frequently center on the early grammatical analysis performed on the incoming signal as it is encoded in memory. For example, Chomsky (1965) writes: ". . . we must add other conditions on the perceptual device beyond mere limitation of memory. We might assume for example that the perceptual device has a stock of analytic procedures available to it, one corresponding to each type of phrase, and that is it organized in such a way that is is unable (or finds it difficult) to utilize a procedure $\phi$ while it is in the course of executing $\phi$ [p. 14]." Similarly, Miller \& Isard (1964) advance an explanation based on a computer analogy. This model, too, seems to be most applicable during the initial analysis of the incoming signal: "... suppose that, while the subroutine (for analyzing relative clauses) is being executed, a second such construction is encountered, so the subroutine is required to call itself. If this recursive feature were not available, confusion would result;... [p. 300]."

The general impression to be gained from these discussions is that the difference in recallability of embedded sentences relative to nonembedded sentences is the result of an incomplete grammatical analysis of the incoming string in the former. This imperfect analysis would involve, among other things, the failure to specify correctly various grammatical relationships, some of which would be necessary for apprehension of meaning. For example, from Example 1 above, it might be hypothesized from these models that the subject noun phrase-verb relationship holding between friend and liked might be weak if it were encoded at all. From the same example, the $\mathrm{NP}_{\text {subject }} \cdot \mathrm{V}$ relationship between lawyer and soaked might also be perceived inf requently and therefore not be seen in attempts to recall. The point here is that these associations, important for meaning, are weak. In short, recall of embedded constructions should show a deficiency in comprehension of these sorts of grammatical relations, and this deficiency is presumably attributable to an 
inability to analyze the incoming string into its meaningful grammatical relationships, i.e., a perceptual deficiency.

Another possible source of the difficulty associated with embedded sentences is in the memory store itself or in the response selection/execution process which utilizes the information stored in memory. That is, it could be argued that difficulties in storing correctly analyzed information, retrieving this information from memory, or formulating a response could account for the observed differences in ability to correctly recall the more complex sentences. Mehler (1963), for example, argued that sentences are stored as deep structure strings with appended lists of grammatical "footnotes" which designate the transformations that must be applied in order to generate the surface structure of the original string. He held that these footnotes (some of the grammatical information) were forgotton at a more rapid rate than were the deep structure strings. In sum, he suggested that the basic grammatical relationships between constituents (e.g., subject, object) necessary for comprehension of meaning were stored more efficiently than the information designating which "grammatical niceties" produce the exact surface structure of the stimulus sentence. On this view, it would be suggested that differential forgetting rates (or interference) might be responsible for the observed differences in difficulty between embedded and nonembedded sentences.

Sacks (1967) and Begg (1971) have further developed this line of reasoning. They find that "... wording information is stored for a very short time ... after which wording memory is a process of reconstructions [Begg, 1971, p. 177]." In effect, this model suggests that differences in reconstruction of surface structure strings from the encoded deep structure strings might account for the difference between embedded and nonembedded sentences.

The foregoing discussion makes it clear that there are several possible loci of the difficulty associated with embedded sentences. The purpose of the present experiment is to ascertain whether the inaccuracy in repeating embedded sentences verbatim is due to incorrect or incomplete information being stored in memory or whether the difficulty lies in retrieving information and/or response execution. In this study, a recall vs recognition paradigm is employed: Ss hear embedded and nonembedded sentences and attempt to either imitate those sentences verbatim or recognize sentences having same/different meanings and different grammatical structures. If the expected difference between embedded and nonembedded sentences is found in recall and a proportional difference is found in the recognition task, this would be consistent with the type of explanatory model suggested by Chomsky and by Miller and Isard. That is, if the difference between embedded and nonembedded sentences is found in both tasks, it could be argued that the different degrees of completeness of grammatical analysis and the differential adequacy of the grammatical specifications of the two sentence types entered into memory underlie performance on both tasks. If, however, embedded sentences are found to be more difficult only in the recall task, an explanatory model involving performance rather than perceptual factors is indicated. That is, if the information stored in memory is sufficiently detailed to give high and equal levels of performance for both sentence types in the recognition task, it cannot be argued that the degenerate quality of this same information underlies the difference between the two sentence types in the recall task. Other factors, such as retrieval, reconstruction of the surface structure from the deep structure, or response execution, would be more likely explanatory concepts.

\section{METHOD \\ Subjects}

Twenty-seven undergraduate volunteers served as Ss. There were 14 females and 13 males.

\section{Sentence Materials}

Sixteen sentences were constructed; each sentence consisted of a matrix sentence and two relative clauses. In eight of the sentences, the two relative clauses were nested and embedded within the matrix, as in Example 1 above. In the remaining eight sentences, the two relative clauses followed the matrix sentence (a configuration termed not nested, not embedded), as in Example 4 above.

The sentences were constructed by selecting randomly from a list of human nouns and active verbs. The nouns and verbs were known to be nonassociates. Each noun and each verb was used twice in the total set of sentences.

All sentences were recorded with normal reading inflection and stress. Examples of the two sentence types were randomly intermixed in the recording.

\section{Procedure}

Two separate studies were run; in Study 1,14 Ss participated, and in Study 2, 13 Ss participated.

\section{Study 1: Recall}

In the recall study, Ss were tested individually. Each sentence in the list was presented twice in immediate succession, with a 3-sec interpresentation interval. Five seconds after the second presentation, a signal was given and $S$ attempted to recall the sentence, exactly as it had been given on tape.

Scoring of recall attempts was directed as much as possible to ascertaining the degree of S's understanding of the target sentences. To this end, the proportion of correct NP-V sequences contained in each recall attempt was tallied. These could be of two forms: $\mathrm{NP}_{\text {subject }}-\mathrm{V}$ or $\mathrm{V}-\mathrm{NP}_{\text {object }}$. For each of the target sentences, six such relations were possible, i.e., two from the matrix sentence, two from the first constituent clause, and two from the second constituent clause. For $S$, the porportions of correctly recalled grammatical relations for all exemplars of $\mathrm{N} / \mathrm{E}$ sentences were averaged and, likewise, a within-S average proportion was computed for NN/NE sentences. These were then averaged across Ss.

\section{Study 2: Recognition}

In the recognition task, the same sentences were employed as were used in the recall task. Each sentence was presented twice, with an interpresentation interval of $3 \mathrm{sec}$. Upon hearing the response signal, which followed the second presentation of a target sentence by $5 \mathrm{sec}$, Ss turned a blank page in a test booklet and silently read a test sentence. The grammatical form of all 
test sentences was the same, irrespective of the grammatical form of the target sentence. That is, all test sentences had a grammatical structure termed long, separated clauses. The following example depicts this form:

(5) The lawyer whom the attendant painted soaked the friend who liked the teller

Sentences of this form may be constructed, containing the same information as is contained in Example 1 (N/E) and Example $4(\mathrm{NN} / \mathrm{NE})$. Half the test sentences constructed for each type of target sentence retained the same basic information as the target sentence. The remaining half of the test sentences were semantically altered such that the meanings of test and target sentence were not equivalent. This was accomplished by transposing either a randomly selected pair of nouns or a pair of randomly selected verbs. The following item would constitute a meaning change item in the sense that it does not preserve the basic relationships expressed in Examples 1,4 , and 5 .

(6) The lawyer whom the friend painted soaked the attendant who liked the teller

The Ss were instructed to read the test item carefully and to indicate on an answer sheet whether the test sentence had the same meaning or a different meaning from the corresponding target sentence. The proportion of correct choices was recorded separately for both types of target sentence.

\section{RESULTS AND DISCUSSION}

The outcome of the recall experiment is simply told: the mean proportion of correct NP.V or V-NP relations recalled for N/E sentences was .15, and the corresponding figure for NN/NE sentences was .44. A within-Ss analysis of differences revealed that the N/E sentence recall was significantly inferior to recall of $\mathrm{NN} / \mathrm{NE}$ sentences $(\mathrm{T}=6.5 . \mathrm{p}<.01)$. This finding replicates and extends the result of Baird (1973) and Miller \& Isard (1963): embedded sentences are more difficult to recall than are not-embedded sentences, even when the recall attempts are scored for meaning retained.

The recognition experiment results, too, can be simply expressed: the mean proportion of correct recognition responses for $\mathrm{N} / \mathrm{E}$ sentences was .69 and for NN/NE sentences, .75. This difference was not significant $[\mathrm{t}(12)=1.10, \mathrm{p}>.10]$. This finding contrasts sharply with the easily demonstrable difference between N/E and NN/NE sentences in recall type tasks. It is the meaning of this difference between results obtained in different tasks to which subsequent discussion will be directed. Before investing effort in explanation, it would be wise to obtain the maximum possible assurance that the recognition results are genuine and replicable. Two pieces of evidence are relevant to this enterprise.

First, these results are due neither to ceiling nor floor effects: the average proportion of correct recognitions for both sentence types are (a) significantly above chance (0.5), and (b) significantly below perfect performance $[\operatorname{ts}(12)>2.21, \mathrm{p}<.05$, in all four comparisons].
It is reasonable, therefore, to rule out the possibility that these results derive from an artifactual restriction of range of possible scores.

Secondly, the failure to obtain the expected sentence-type differences in a recognition paradigm was replicated. In an independent study, 12 undergraduate volunteers were asked to say whether word sequences four words in length had or had not been drawn from a sentence just heard. The target sentences were N/E and $\mathrm{NN} / \mathrm{NE}$ constructions. Half the four-word sequences were taken directly from target sentences, and half were constructed by selecting a four-word string and transposing two adjacent words. A second group of 12 Ss provided empirical estimate of chance performance.

The recognition scores for $\mathrm{N} / \mathrm{E}$ and $\mathrm{NN} / \mathrm{NE}$ sentences in this experiment (after "chance" successes were subtracted out) were 4.21 and 4.25 (Wilcoxon $\mathrm{T}=38$, $\mathrm{p}>.10)$.

It is a truism that replication of a null result is itself meaningless. However, taken in context, the absence of $\mathrm{N} / \mathrm{E}-\mathrm{NN} / \mathrm{NE}$ differences in recognition is meaningful. It suggests that within the range of complexity and length of sentence employed in these studies, the meaning of $\mathrm{N} / \mathrm{E}$ and NN/NE sentences is apprehended, encoded, and retained to the same extent. It cannot be argued, therefore, that the N/E-NN/NE differences consistently obtained in recall are attributable to Ss' relative inability to correctly encode the meaning of the incoming $\mathrm{N} / \mathrm{E}$ sentences.

Several other alternative "explanations" are consistent with these data. For example, verbatim recall demands encoding of sufficient grammatical information to enable one to reconstruct the surface structure of the target sentence. It is possible that encoding of this information is difficult, or that this information is more rapidly lost than are meaning relations (cf. Sacks, 1967; Begg, 1971). The results of the experiments reported above do not permit one to distinguish between these and other alternative hypotheses. The significance of these results is primarily negative, in the sense that these data reveal the inadequacies of current accounts of processing complex sentences, and compel further refinement of the models.

\section{REFERENCES}

Baird, R. Structural characteristics of clause-containing sentences and imitation by children and adults. Journal of Psycholinguistic Research, 1973, 2, 115-127.

Begg, I. Recognition memory for sentence meaning and wording. Journal of Verbal Learning \& Verbal Behavior, 1971, 10, 176-181.

Chomsky, N. Aspects of the theory of syntax. Cambridge, Mass: M.I.T. Press, 1965.

Mehler, J. Some effects of grammatical transformations on the recall of English sentences. Journal of Verbal Learning \& Verbal Behavior, 1963, 2, 346-351.

Miller, G. A., \& Isard, S. Free recall of self-embedded English sentences. Information \& Control, 1964, 7, 292-303.

Sachs, J. S. Recognition memory for syntactic and semantic aspects of connected discourse. Perception \& Psychophysics, $1967,2,437-442$.

(Received for publication September 24, 1973). 